\title{
The model organism diaspora
}

\section{Jonathan Hodgkin ${ }^{1}$}

Received: 23 November 2018 / Accepted: 20 December 2018

(c) The Genetics Society 2019
The 1950s and 1960s saw an extraordinary flowering of molecular biology and molecular genetics, which revealed many of the fundamental secrets of life. After that era, many of the scientists who had contributed to this Golden Age (no exaggeration) of discovery looked around for new worlds to conquer and new experimental systems to develop. It was obvious that genetic approaches would provide powerful tools, especially for investigating complex biological phenomena. Francis Crick once accurately remarked, "Genetics is great for opening up a problem and fantastic in the endgame, but in the middle you have to do biochemistry". For many, it is the first of these phases that is most rewarding, but it is still crucial to choose the right system for your preferred problems, not least because of the need to do at least some biochemistry.

Consequently, in the space of a decade or so, an intellectual diaspora of gifted molecular geneticists took place, as these scientists chose a variety of different organisms for their research. Max Delbrück began to work on Phycomyces, Seymour Benzer and David Hogness on Drosophila, Sydney Brenner on nematodes, Francois Jacob on mice, George Streisinger on zebrafish, Gunther Stent on leeches, Cyrus Levinthal on Daphnia, Gerry Fink on Arabidopsis, Bill Dove on Physarum, Dale Kaiser on myxobacteria, Lee Hartwell and Ron Davis on yeast, and so on. Some of these organisms, such as Drosophila and Arabidopsis, had been studied for decades by conventional genetic methods, but it was a more open question as to how amenable they might be to molecular approaches.

Some of these adventures worked out spectacularly well and some did not, for a variety of reasons. One example of an interesting organism that never managed to attract a large number of researchers was Delbrück's choice, the fungus

Jonathan Hodgkin

jonathan.hodgkin@bioch.ox.ac.uk

1 Department of Biochemistry, University of Oxford, South Parks Road, Oxford OX1 3QU, UK
Phycomyces blakesleeanus, described by him as "the most intelligent primitive eukaryote" (cited in Fischer and Lipson 1988). Its sporangiophores exhibit an extraordinary range of sensory abilities, including a still rather mysterious avoidance behaviour whereby the growing sporangiophore is able to avoid nearby objects (Cerdá-Olmedo 2001). Delbrück was originally a physicist, and hoped to discover new kinds of physics by investigating biology, so the unusual avoidance phenomenon may have piqued his interest. Research on Phycomyces was unfortunately hampered by the difficulties of its sexual system and by inability to achieve transformation with exogenous DNA. Delbrück was also avowedly reluctant to get involved with biochemical details (Strauss 2017).

In contrast, Delbrück's previous choice of an experimental system, the $\mathrm{T}$ bacteriophages of Escherichia coli, had been brilliantly successful. He realized in 1939 that phage provided an ideal route to understanding the fundamental nature and properties of genes, as the subsequent history of the "Phage Group" abundantly demonstrated (Ellis and Delbrück 1939). However, in the longer run T4, the most popular of these bacteriophages, proved to have a significant defect as an experimental system. The DNA of T4 is heavily glucosylated, rendering it resistant to most restriction enzymes and therefore complicating the application of many of the techniques of molecular genetics. This difficulty could not have been foreseen. Sometimes, unexpected problems are there from the outset, one example being Gregor Mendel's unlucky decision to test the theories that he had developed by breeding garden peas, by experimenting on a different plant. He chose hawkweed, not knowing that these plants reproduce asexually and are also highly heterozygous, so his breeding experiments produced incomprehensible results (Nogler 2006).

As well as problems, major unforeseen advantages may also become apparent when a new system is being developed, sometimes from the general investment of experimental effort and sometimes from sheer good fortune. One spectacular early example was the discovery of polytene chromosomes in the salivary glands of Drosophila (Painter 
1933), some 20 years after T. H. Morgan had decided on fruit-flies as his preferred genetic system. Polytene chromosomes added a huge bonus to research on Drosophila, as they provided a means of directly visualizing fine details of chromosome structure and thereby permitted far more sophisticated cytogenetics than was possible in other genetic organisms. Polytene chromosomes continued to be highly advantageous for decades, especially in the era of molecular gene cloning, with the development of in situ hybridization.

However, Nature's gift of polytene chromosomes did have one unhelpful consequence, arising from detailed mutational analyses of defined intervals in the Drosophila genome, which led to the beguiling "one chromomere, one cistron" hypothesis (Judd et al. 1972). The possibility that each visible polytene chromosome band or chromomere corresponded to an individual gene was exciting and generated much theorizing as to the nature of the eukaryotic gene. This was a major issue for many years, partly triggered by the $\mathrm{C}$ value paradox: most eukaryote genomes contain far more DNA than bacterial genomes, suggesting that eukaryote and prokaryote genes might be different in some fundamental way. Sadly, the correspondence between genes and bands in Drosophila evaporated over time, and ultimately proved to be no more than accidental.

A much later example of good fortune was the discovery of feeding RNA interference (RNAi) in the nematode Caenorhabditis elegans (Timmons and Fire 1998). This species takes up exogenous double-stranded RNA from the environment with much greater efficiency than its close relatives, thereby vastly simplifying the application of whole-genome RNAi screens. Caenorhabditis elegans had already proved to be a fortunate choice in other ways, for example, in its optical properties and in the ease with which it can be frozen and stored indefinitely.

So, some model organisms seem to be blessed, others not so much. A variety of strategic factors influenced early choices in the 1960s, and it was always clear that no single organism could satisfy all requirements. These factors included ease and expense of culture, size, complexity, transparency, growth rate, generation time, genetic amenability, long-term storage, behavioural repertoire, natural variation, background knowledge, phylogenetic position, scientific community, and medical or economic relevance. Some of these factors significantly influenced funding decisions. Moreover, there were inevitable trade-offs in experimental advantages, such as those encapsulated in Quinn's Law: "The convenience of genetics multiplied by the quality of the behavioural repertoire is a constant for all animals" and Gould's Corollary: "The quality of the genetics multiplied by the ease with which single nerve cells can be identified and penetrated is also a constant" (Quinn and Gould 1979).
In subsequent decades, other issues became important, notably the ability to clone genes, to transform the organism, and eventually to generate physical maps and complete sequences of the genome. Genome size was and remains an important factor in experimental tractability, from this point of view. Both C. elegans and Arabidopsis thaliana were found to have very compact genomes (100 and $135 \mathrm{Mb}$ respectively), which made them significantly more attractive for genomic approaches. Towards the other end of the scale, the zebrafish Danio rerio has a genome of $1400 \mathrm{Mb}$, and obtaining a satisfactory complete genome sequence for this organism took many years of effort.

The most popular model organisms all had multiple advantages and thereby lent themselves to many different kinds of research. In contrast, some organisms have special properties that rendered them ideal for certain experimental projects, illustrating the principle enunciated by August Krogh (Krogh 1929), and before him by Claude Bernard (Bernard 1865). The protist Tetrahymena, for example, provided a perfect system for the study of telomeres, because the generation of its macronucleus entails fragmentation of its genome into hundreds of minichromosomes and the creation of correspondingly many telomeres. Another genetically amenable protist, Paramecium, has both interesting behaviour and cells large enough to permit microelectrode penetration and electrophysiological experiments, thereby permitting the first genetic analyses of ion channels.

Efficient gene cloning and ease of transformation became major factors in determining the popularity of research organisms. Identification and characterization of transposons, as tools for molecular genetics, also became important. Antirrhinum majus (snapdragon), for example, is not obviously optimal experimental material, but its Tam transposons enabled the ready isolation of floral homeotic mutants and the pioneering elucidation of flower morphogenesis by Enrico Coen and collaborators (Carpenter and Coen 1990).

The question of what genetic system to choose was one that came up frequently in my own research experiences in the 1970s. I was fortunate enough to carry out my doctoral research at the MRC Laboratory of Molecular Biology (LMB) in Cambridge, working on the nematode worm $C$. elegans under the supervision of Sydney Brenner, sometimes known as "Father of the Worm" for his role in pioneering the use of $C$. elegans. He attracted and inspired many talented students and postdoctoral scientists who went on to found their own research groups and pursue illustrious careers. Most of these had previous experience in the classical systems of molecular genetics (bacteriophage, bacteria or fungi), and like their mentors had wondered what system to work with next. The worm looked like an appealing but initially risky possibility. Nevertheless, 
several more senior and established investigators were also recruited.

For myself, after 3 years working with $C$. elegans, I wanted to try something different and spent a couple of years at Stanford working in Dale Kaiser's lab. Dale was one of heroes of research on bacteriophage lambda, which I found utterly fascinating, but he too had by then (1974) moved on to something different: myxobacteria, which had the advantage of being both multicellular and bacterial (Kaiser et al. 1979). But it was also a novel system, and therefore sometimes frustrating. Nevertheless, we made progress. I was tempted to stay at Stanford and continue with Myxococcus xanthus, but Brenner and Crick had offered me a research staff position at LMB and it was not clear how long that offer would stay open. Moreover, Brenner said that I could work on anything I liked at LMB, short of "studying why the birds sing in the trees". He may have recalled that my final year undergraduate research project had involved studying the behaviour of rooks in the countryside around Oxford. At the time, I wrote to him that mice looked like ultimately the best system to work on. Nevertheless, starting on mouse genetics would have been daunting, despite the hints of new inroads into developmental biology. Something like the mouse t-complex looked important and exciting. However, it was already becoming clear that the accumulation of interesting developmental mutations in the t-complex was most probably a sort of genetic artefact, which was a discouraging realization.

So the question was, what were the most promising systems, and what really important questions could be attacked productively? What else was out there? Back in Cambridge, I spent some time experimenting with Halobacterium halobium, because Richard Henderson and Nigel Unwin had just produced their historic first structure for its purple membrane protein, bacteriorhodopsin (Henderson and Unwin 1975), and Archaea such as Halobacterium represented virgin territory for bacterial genetics. Unfortunately, it became clear that Halobacterium strains were appallingly unstable from a genetic point of view, and therefore going to be hard to work with. An alternative was Arabidopsis, which looked tempting. So did Drosophila. However, both the weeds and the flies had generation times substantially longer than that of $C$. elegans, and therefore lacked the gratification provided by worms, of getting new results almost every day. Brenner used to claim that geneticists chose organisms with generation times proportional to the speed of their own thought processes. This was scarcely fair to those who worked on maize, which has only one or two generations a year, yet yielded profoundly important results in the hands of Emerson, McClintock, Rhoades, Beadle and others. However, there is much to be said for being able to do genetic crosses in days rather than months. Among other things, if you are going wrong, you find out faster.

So, short generation times and compact genomes are unquestionably convenient from a technical point of view, but here again there are downsides, for example, in grand phylogenetic comparisons and studies of evolution. We know now that Saccharomyces, Drosophila and $C$. elegans have all lost gene families and genetic features that are important in mammals and were also present in ancestral eukaryotes, because they can still be found in the genomes of organisms such as the protist Naegleria gruberi (FritzLaylin et al. 2010) or the cnidarian Nematostella vectensis (Matus et al. 2007). At least some of these losses probably arise from the increased number of generations that the fastbreeding genetic models have gone through, relative to other organisms.

However, this disadvantage was not a consideration in the 1970s. At that time, research on C. elegans at LMB and elsewhere was expanding rapidly. John Sulston and Bob Horvitz had worked out the postembryonic cell lineages of $C$. elegans and begun to investigate cell lineage mutants. Bob Horvitz had also extended some of my own thesis work on meiotic mutants, and we collaborated on a more complete analysis of these. Other parts of my thesis work, on sex determination and sex-specific developmental mutants, had become more promising and exciting as a result of the cell lineage work. Moreover, the "worm breeders" had by then grown into a remarkably congenial international research community, thanks in particular to Bob Edgar (another T4 veteran, like Horvitz and Brenner himself). The easiest and most productive path for me was to drift back into full time research on $C$. elegans, which is a decision I have rarely regretted.

In subsequent decades, the proven value of model organism research has led to yet further choices of plants or animals for intensive experimentation-for example, the moss Physcomitrella patens, the flatworm Schmidtea mediterranea, the nematode Pristionchus pacificus and the beetle Tribolium castaneum have all acquired substantial bodies of information and technical experience. With the availability of ever cheaper DNA sequencing, and tools such as RNAi and CRISPR/Cas9, it has become possible to contemplate an even wider set of organisms for detailed investigation and manipulation (Goldstein and King 2016).

A final aspect of these explorations, which have sampled a great variety of organisms, was the broadened attention paid to the whole panorama of life on earth at a molecular level. Most of the experimental systems were chosen for their usefulness as general models, but they have also provided much valuable information about the special features of their particular taxonomic groups: Drosophila for insects, C. elegans for nematodes, Saccharomyces cerevisiae for fungi, and so on. Such information has great and 
continuing relevance to the economic, agricultural and medical applications of basic research, and these practical applications will undoubtedly continue and increase.

\section{Compliance with ethical standards}

Conflict of interest The author declares that they have no conflict of interest.

Publisher's note: Springer Nature remains neutral with regard to jurisdictional claims in published maps and institutional affiliations.

\section{References}

Bernard C (1865) Introduction à l'étude de la médecine expérimentale. Flammarion, Paris

Carpenter R, Coen ES (1990) Floral homeotic mutations produced by transposon-mutagenesis in Antirrhinum majus. Genes Dev 4:1483-1493

Cerdá-Olmedo E (2001) Phycomyces and the biology of light and color. FEMS Microbiol Rev 25:503-512

Ellis EL, Delbrïck M (1939) The growth of bacteriophage. J Gen Physiol 22:365-384

Fischer EP, Lipson C (1988) Thinking about Science. Max Delbrück and the origins of Molecular Biology. WW Norton and Company, New York

Fritz-Laylin LK, Prochnik SE, Ginger ML, Dacks JB, Carpenter ML, Field MC, Kuo A, Paredez A, Chapman J, Pham J, Shu S,
Neupane R, Cipriano M, Mancuso J, Tu H, Salamov A, Lindquist E, Shapiro H, Lucas S, Grigoriev IV, Cande Z, Fulton C, Rokhsar DS, Dawson SC (2010) The genome of Naegleria gruberi Illuminates early eukaryotic versatility. Cell 140:631-642

Goldstein B, King N (2016) The future of cell biology: emerging model organisms. Trends Cell Biol 26:818-824

Henderson R, Unwin PN (1975) Three-dimensional model of purple membrane obtained by electron microscopy. Nature 257:28-32

Judd BH, Shen MW, Kaufman TC (1972) The anatomy and function of a segment of the $\mathrm{X}$ chromosome of Drosophila melanogaster. Genetics 71:139-156

Kaiser D, Manoil C, Dworkin M (1979) Myxobacteria: cell interactions, genetics, and development. Annu Rev Microbiol 33:595-639

Krogh A (1929) The progress of physiology. Am J Physiol 90:243-251

Matus DQ, Magie CR, Pang K, Martindale MQ, Thomsen GH (2007) The Hedgehog gene family of the cnidarian, Nematostella vectensis, and implications for understanding metazoan Hedgehog pathway evolution. Dev Biol 313:501-518

Nogler GA (2006) The lesser-known Mendel: his experiments on Hieracium. Genetics 172:1-6

Painter TS (1933) A new method for the study of chromosome rearrangements and the plotting of chromosome maps. Science 78:585-586

Quinn WG, Gould JL (1979) Nerves and genes. Nature 278:19-23

Strauss BS (2017) A physicist's quest in biology: Max Delbrück and "complementarity". Genetics 206:641-650

Timmons L, Fire A (1998) Specific interference by ingested dsRNA. Nature 395:854 